\title{
A Dense Magnetic Coil Array with Multi-locus and Multi-site Patterning
}

This paper was downloaded from TechRxiv (https://www.techrxiv.org).

LICENSE

CC BY-NC-SA 4.0

SUBMISSION DATE / POSTED DATE

$11-11-2021 / 12-11-2021$

CITATION

Smith, Matthew; Sievenpiper, Daniel (2021): A Dense Magnetic Coil Array with Multi-locus and Multi-site Patterning. TechRxiv. Preprint. https://doi.org/10.36227/techrxiv.16992559.v1

$\mathrm{DOI}$

10.36227/techrxiv.16992559.v1 


\title{
A Dense Magnetic Coil Array with Multi- locus and Multi-site Patterning
}

\author{
Matthew C. Smith, Sr. Member, IEEE and Daniel F. Sievenpiper, Fellow, IEEE
}
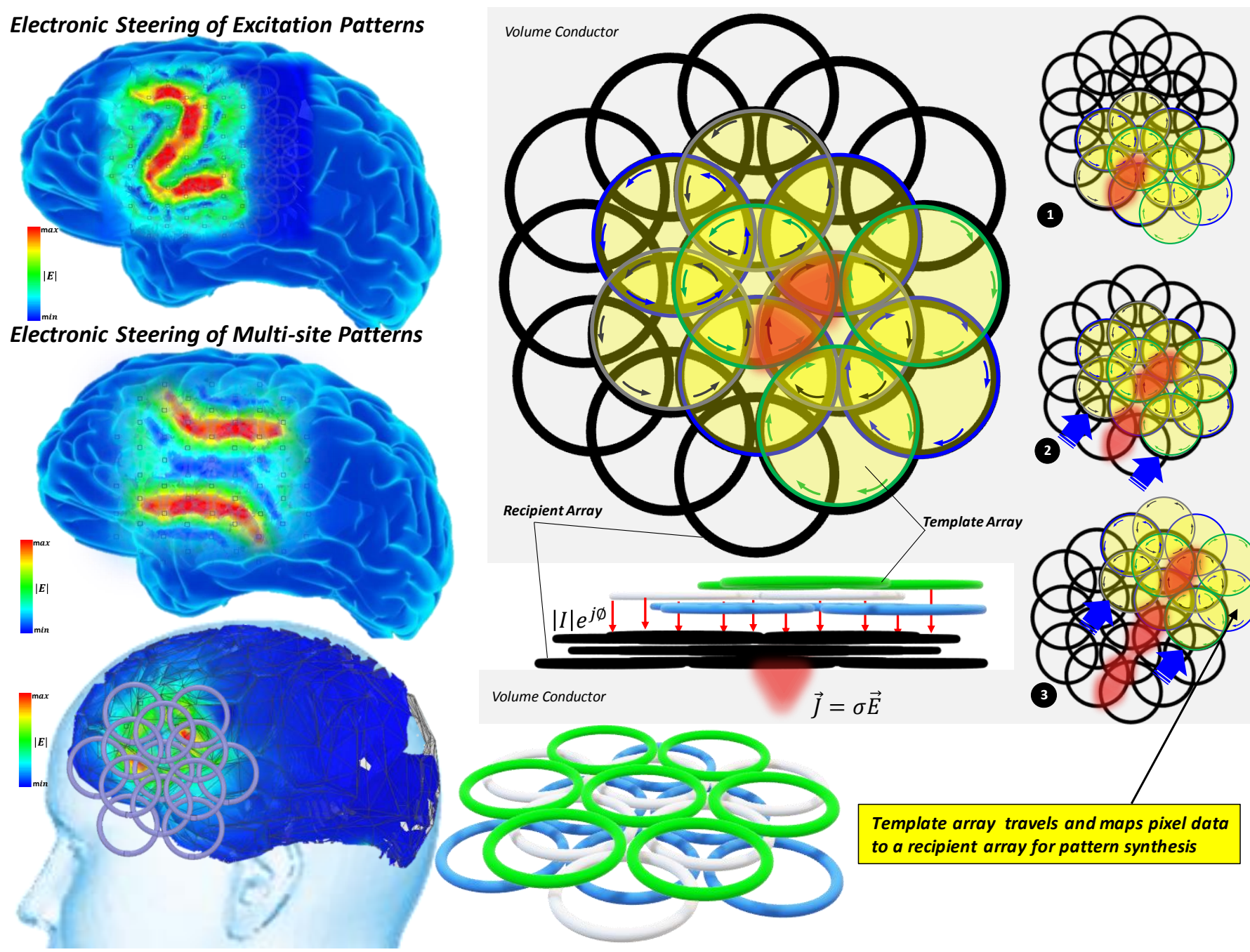

Multi-loci and multi-site spatiotemporal stimulation and excitation is enabled by the 3-layer dense array.

Take-Home Messages

- We propose multi-loci and multi-site current patterning (sequential or simultaneous) for precise, rapid and repeatable steering for improved neuronal targeting.

- Here we demonstrate these improvements by using a unique pixel cell for excitation synthesis as enabled by a three-layer dense magnetic coil array that is mapped to a hexagonal grid with cubic coordinates.

- We validate the theory with supporting simulations, experimental results and a scalable electronics design that can address a relevant range of larger coil diameters, higher power levels and topologies.

- Precise, rapid and repeatable patterns potentially offer new modalities for noninvasive neuromodulation (suprathreshold and subthreshold) and adjacent biomagnetic applications such as tumor cell membrane disruption [1], and magnetic drug delivery [2]. 


\title{
A Dense Magnetic Coil Array with Multi-locus and Multi-site Patterning
}

\author{
Matthew Charles Smith, Senior Member, IEEE, and Daniel F. Sievenpiper, Fellow, IEEE
}

Department of Electrical and Computer Engineering, University of California San Diego, La Jolla, CA 92093, USA

\begin{abstract}
Noninvasive biomagnetic neuromodulation systems have, for over three decades, produced a rich foundation of contributions to neurological research and therapies. New advancements such as improved precision in targeting, repeatability, and multi-locus spatiotemporal stimulation are capabilities that are sought after by this community. In this regard, we propose potential advancements using a dense magnetic coil array with multiple modalities for improved targeting in and around a target site and multi-locus current patterning (sequential or simultaneous). We demonstrate, through simulations and experimental data, that these capabilities are possible using a new fundamental unit, the pixel cell, for pattern synthesis. A unique method of pattern synthesis is described that transfers user-defined currents and phases from a template array to an underlying recipient array for the construction of an excitation pattern. Hexagonal grids and are mapped to cubic coordinates for efficient pattern formation. These findings offer potentially useful fundamental research contributions towards developing new techniques for improved precision and rapidly executed spatiotemporal modalities over that of manual or robotically steered single function systems. The system architecture was designed to be a fundamental research testbed with electronics and coils that are scalable to higher power levels and adaptable to a variety of biomagnetic applications in noninvasive neuromodulation (suprathreshold and subthreshold) magnetic and adjacent applications in magnetic drug delivery, and tumor cell membrane disruption.
\end{abstract}

Index Terms - magnetic coil array, multi-site excitation, near-field biomagnetics, hexagonal grids, cubic coordinates, noninvasive neuromodulation, multichannel transcranial magnetic stimulation

\section{INTRODUCTION}

$\mathrm{I}$ MPROVED capabilities in biomagnetic neuromodulation such as precision and repeatability in targeting, multi-site spatiotemporal loci, and reduced extraneous excitation are being actively researched by the neuroscience community [3][5]. To that end, the main objective of our study involves developing a fundamental research testbed that can contribute to such improvements. Our system is unique in that intersecting coils in a three-layer array form a discrete triangular intersection region, a Reuleaux triangle [6], coined the pixel cell, that induces electric fields (E-fields) and eddy currents on a hexagonal grid mapped to cubic coordinates. Bio-magnetic stimulation uses a time varying magnetic field, created by a pulsed current, through a coil that induces an E-field and eddy currents in a conductive medium. This results in a total induced E-field with two components. A primary E-field is created by the coil and a secondary E-field is created by changes in conductivity along the current path resulting in a nonuniformity of charges [7]. This total E-field excites or inhibits neuronal action potentials. Transcranial Magnetic Stimulation (TMS), is the exemplar of nonsurgical neuromodulation today [8] with a figure-8 coil to enhance the focality [9] and when deeper penetration is required, a larger diameter coil is used at the expense of focality [10],[11]. Typically, these are single function systems with one coil that is manually or robotically positioned [12] over a target excitation site. In contrast, multichannel, multi-coil arrays [13] have the potential in one multifunctional system to offer improvements via precise, rapid

The authors are with the Electrical and Computer Engineering Department, University of California San Diego, La Jolla, CA 92093 USA. (e-mail: mcs008@eng.ucsd.edu and dsievenpiper@eng.ucsd.edu). and repeatable electronic targeting over multiple neuronal sites. Thus, contributing to unexplored areas of research and to augment traditional single-function systems. Despite these benefits, implementation challenges have been identified that include thermal management, coil-to-coil proximity effects, rapid E-field attenuation and extreme Lorentz forces due to the smaller diameter coils $(<30 \mathrm{~mm})$ that were proposed previously [13]. However, recently researchers have developed larger diameter coils at higher current levels with up to five channels [3] to examine these challenges and to explore this concept. We take a different approach with the same goals that leverages our scalable system architecture (electronics and coils) allowing for proof of concepts to be tested initially with lower current levels $(10-40 \mathrm{~A})$ and then moving up to support $>1000 \mathrm{~A}$ with larger wire gauge coils. This allows us to initially focus on multi-locus patterns and multi-site patterns as enabled by more efficient synthesis techniques. A simulation of a curved multi-locus pattern using our approach is seen in Fig. 1.

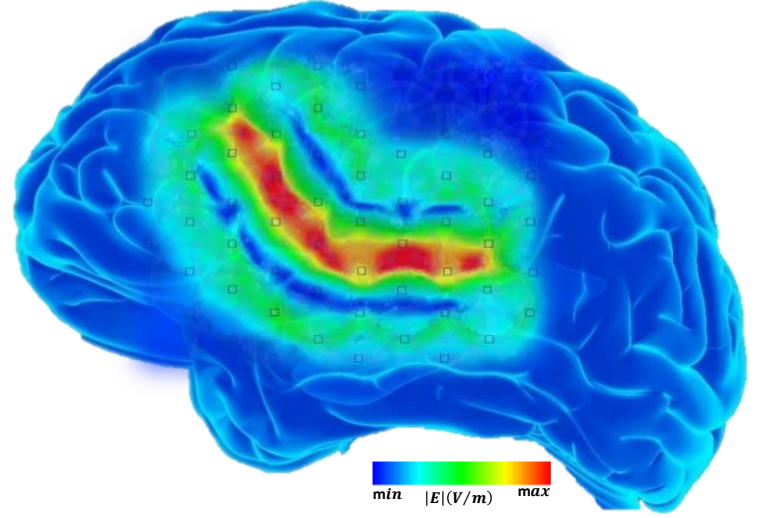

Fig. 1. A curved excitation locus using the dense array is synthesized and patterned orthogonal to several sulci using pixel cell units. 
Typically a single coil topology, single layer array and many existing multi-layer array designs can only generate eddy currents in one direction in each region. With our approach, the direction and magnitude of induced current are independently controlled, within the limits of Maxwell's equations and continuity. Moreover, we can set up arbitrary null areas steering return currents away from areas of unwanted extraneous excitation, a subject of a paper following this one. The proposed array also has the ability to control the direction of the induced eddy currents, which may be useful for future treatments that will take advantage of this additional degree of freedom. For example, creating currents that are either longitudinal or transverse to complex curved structures such as gyri and sulci of the brain which vary from patient to patient. Additionally, user-defined excitation patterns and multiple-loci can be steered over multi-sites (simultaneous or sequentially) that would take several of today's systems to achieve. Movement of single function stimulator coils, in today's systems, makes rapid movement challenging due to heavy robotic arms or manual steering. In contrast, electronic targeting promises to offer rapid site-to-site spatiotemporal switching with precise targeting. Moreover, if simultaneous excitation at multiple sites is desired for connectivity studies between brain regions then multiple single function coil systems have limitations. Electronic switching of multichannel coils thus emerges as a potentially advantageous alternative which merits further fundamental research.

\section{PRINCIPLES OF OPERATION}

The general principles for the synthesis of multi-locus pattern excitation using pixel cells as fundamental units of construction are described herein. At the onset of development, our main objective was to find a coordinate system that could maintain the coil current parameters for all three array layers in one grid and coordinate system. The system also needed to support mathematically efficient movement (rotation and translation) across a given grid in order to facilitate ease of programming in in NumPy [14] the mathematical library within Python. Hexagonal grids with a mapping to cubic coordinates were selected and found to best support these objectives. This general technique is also used by video game designers for animation and landscape construction [15]. Also, axial coordinates, a closely related system to cubic coordinates, was selected to store the coil current data.

\section{Pixel Cell Excitation}

The shape formed by the intersecting coils of three layers is geometrically a Reuleaux triangle [6], coined the pixel cell in Fig. 2. It is defined as the fundamental building element for the synthesis of excitation patterns in the dense array. This triangular region gives rise to primary induced currents in three directions with return currents spread over the volume conductor underneath the array. Our approach allows the direction and magnitude of current in every pixel to be independently controlled, within the limits of Maxwell's equations and continuity.

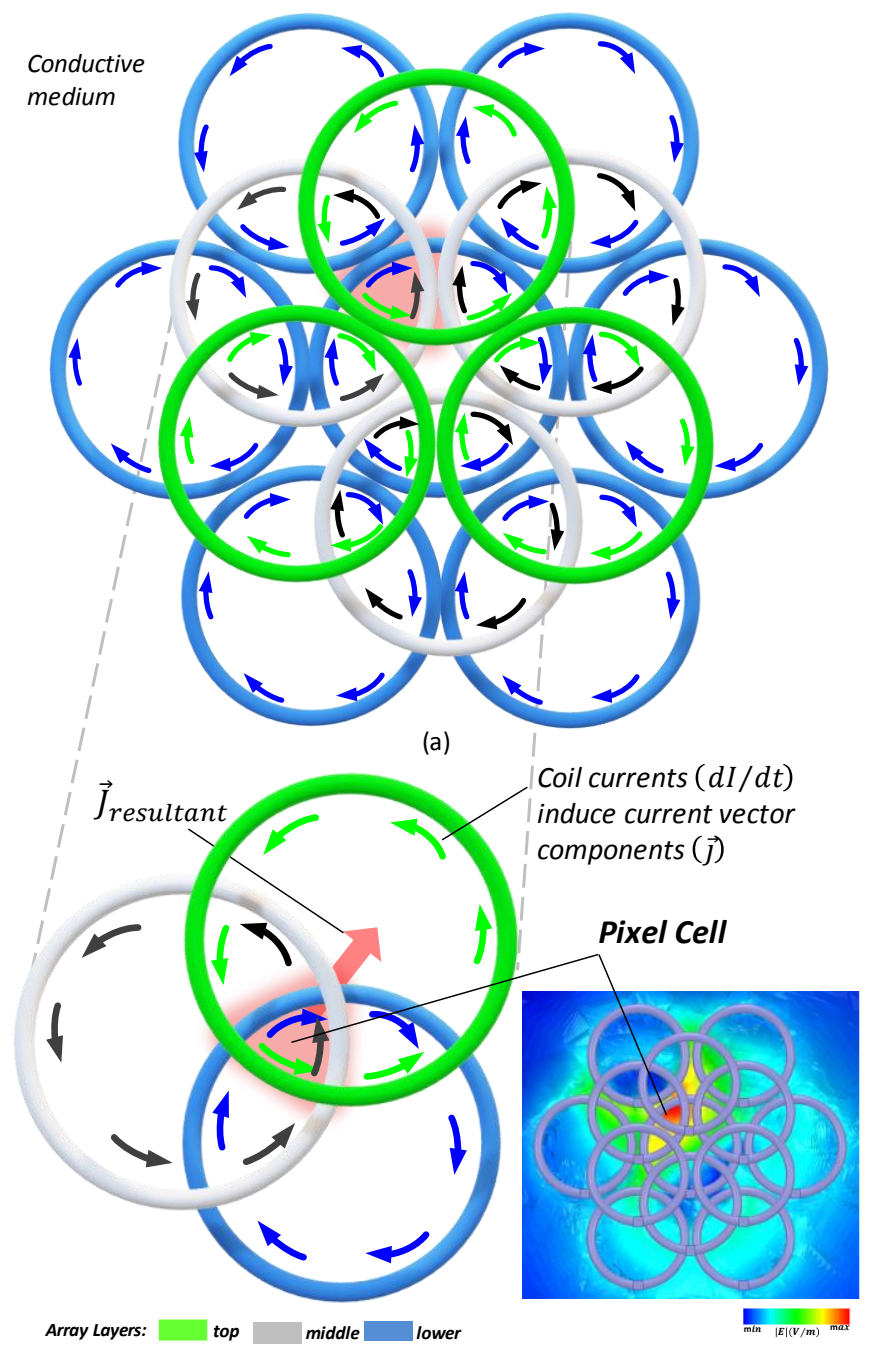

(b)

(c)

Fig. 2. Pixel cell excitation principles with our three-layer array inducing a singular pixel in a conductive medium (note: excitation site in red). (a) 13-coil three- layer array with requisite currents and phase, (b) three overlapping coils form a triangular pixel cell mapped to specific drive coil current magnitude and phase giving rise to a resultant current vector in the pixel cell and, (c) simulation of induced E-field magnitude of a single pixel excitation.

Currents are deemed more tractable when one considers the fact that a time-varying current in a coil creates a magnetic field normal to the coil, which in turn induces a resultant E-field and current density vector in a specific direction in the neuronal target area.

An a priori selected coil current magnitude and phase induces E-field and eddy currents $(\vec{J}=\sigma \vec{E})$ in a conductive medium. The currents induced are a function of the drive current magnitude and phase $(0$ or $\pi)$ in each of the three intersecting coils in Fig 2 (b). The underlying target region, under the excited pixel cell, gives rise to a concentrated E-field simulated in Fig.2 (c).

The minimum addressable area of the pixel is then one sixth (1/6) of the area of a single coil. It follows then that this technique can be scaled up to accommodate much larger coil diameters and wire gauges for high power levels given the appropriate thermal management system. 


\section{Induced Pixel Current Vectors}

Six equilateral triangles cover the area of a coil in Fig. 3 and are assigned to each pixel cell. This facilitates basic current density vector analysis whereby the line segments of the triangle provide the relative vector weighting in each direction that combine to give the resultant vector and current direction for a given pixel. A $30^{\circ}$ tilt of the triangle to the standard reference axis is performed for convenience for each of the pixel variant examples in Fig. 3.

The general function that expresses the relationship between the time-varying current in each of the three layers of coils, the induced E-field and current density vectors in the conductive medium at an excitation pixel is

$$
\vec{J}_{t_{q r}}=j_{t_{q r}} \widehat{x}+j_{t_{q r}} \widehat{y}=\sigma \vec{E}_{t_{q r}}
$$

where $\vec{J}_{t_{q r}}$ is the resultant induced current density vector, $j_{t_{q r}} \hat{x}$ and $j_{t_{q r}} \hat{y}$ are the vector components, $t_{q r}$ is the specific pixel, $\sigma$ is the conductivity ( $\mathrm{S} / \mathrm{m}$ ) of a tissue equivalent solution, and $\vec{E}$ is the induced $\mathrm{E}$-field. The $\mathrm{x}$ and $\mathrm{y}$ axis induced current vector components are then expressed as

$$
\begin{aligned}
& j_{t_{q r} \hat{x}}=\left( \pm\left(\frac{d I_{\text {top }}}{d t}\right) \delta_{x} \pm\left(\frac{d I_{\text {mid }}}{d t}\right) \delta_{x} \pm\left(\frac{d I_{\text {lower }}}{d t}\right) \delta_{x}\right) \hat{x} \\
& j_{t_{q r}} \hat{y}=\left( \pm\left(\frac{d I_{\text {top }}}{d t}\right) \delta_{y} \pm\left(\frac{d I_{\text {mid }}}{d t}\right) \delta_{y} \pm\left(\frac{d I_{\text {lower }}}{d t}\right) \delta_{y}\right) \hat{y}
\end{aligned}
$$

where $d I_{q r} / d t$ is the time-varying current in the coil in a specific array layer (i.e., top, mid and lower) and $\delta$ represents the component vector weight projections predicated on the line segments of the triangle.

Several variants of the pixel cell are characterized in Fig. 3 They are the single excitation pixel, the two-pixel excitation unit and a single null pixel. A single excitation pixel in a 13coil array is illustrated in Fig. 3(a) along with its simulated Efield in Fig. 3(b). An enlarged view of this array's central area, in Fig. 3(c), reveals the requisite coil currents and phases that induce the current density vectors for the pixel $t_{q r}$. Using Eqn. (1), (2) and (3) yields the magnitude and direction of total induced current density vector, $\vec{J}_{t_{q r}}=\hat{x}+\sqrt{3} \hat{y}$ illustrated in Fig. 3(d) (note: unity is used for peak drive coil current magnitudes for simplification).

A two-pixel excitation unit in a 13-coil array is illustrated in Fig. 3(e) along with its simulated E-field in Fig. 3(f). An enlarged view of this array's central coil, in Fig. 3(g), reveals the requisite coil currents and phase vectors that induced the current density vectors for the pixels $t_{q r}$. Using Eqn. (1), (2) and (3) yields the magnitude and direction of total induced current density vector, $\vec{J}_{t_{q r}}=-2 \sqrt{3} \hat{y}$ illustrated in Fig. 3(h) (note: unity is used for peak drive coil current magnitudes for simplification).
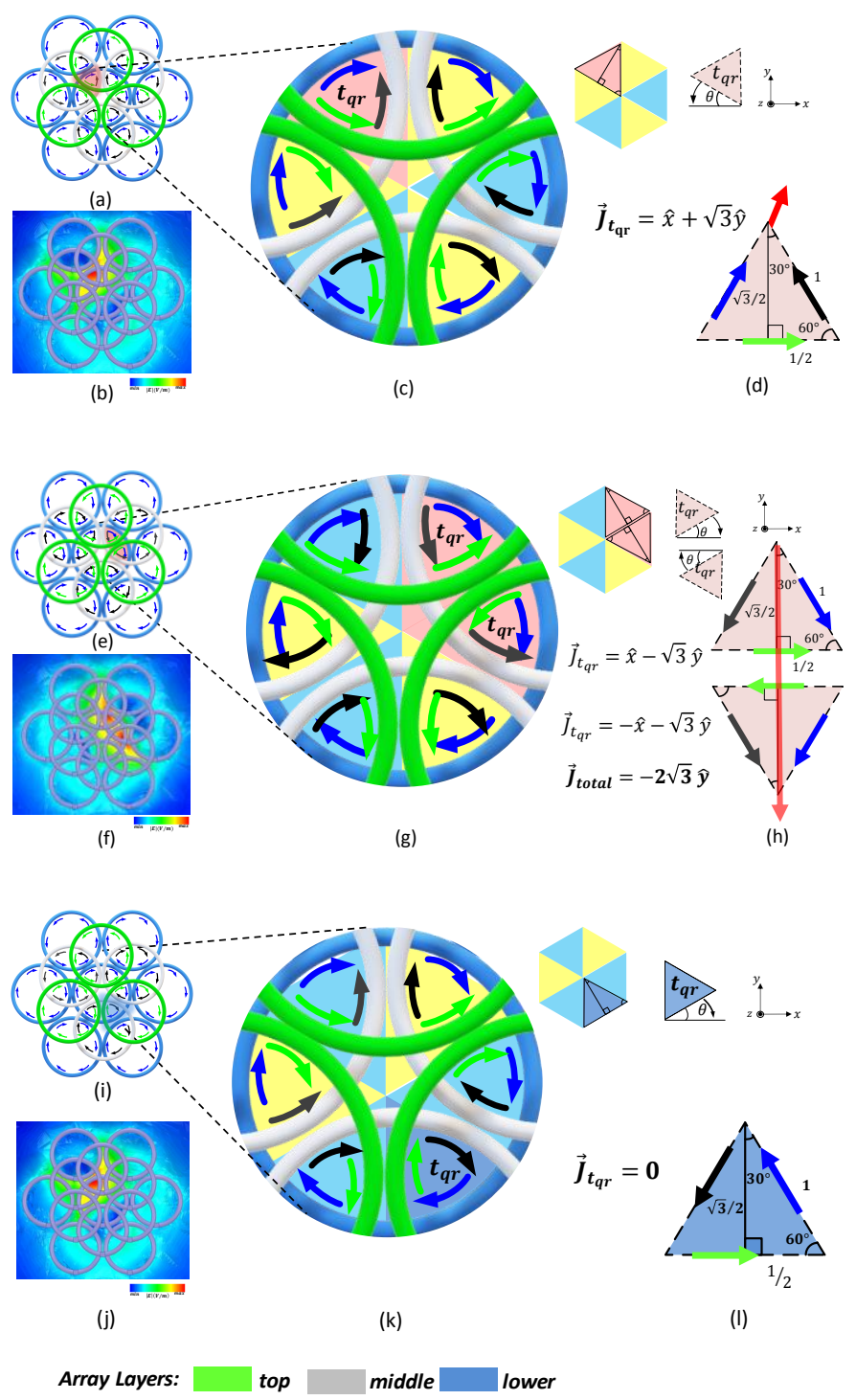

Fig. 3. Three variants of a pixel cell are characterized in a 13-coil array (note: excitation site is depicted in red and null in dark blue). (a) coil layers and current phase to excite a single pixel in a 13-coil array, (b) FEM of E-field of the pixel, (c) enlarged view - center coil and induced current density vectors for the pixel $t_{q r}$, (d) magnitude and direction of total induced current density vector, $\vec{J}_{t q r}=$ $\hat{x}+\sqrt{3} \hat{y}$, (e) coil layers and current phase to excite a single double-pixel in a 13-coil array, (f) FEM of E-field of the double-pixel, (g) enlarged center coil and induced current density vectors for the summing of the two pixel $t_{q r}$, (h) the magnitude and direction of total induced current density vector, $\vec{J}_{t r r}=-2 \sqrt{3} \hat{y}$ , (i) coil layers and current phase to create a single null pixel in a 13-coil array, (j) FEM of E-field of the null pixel, (k) enlarged view of center coil induced current density vectors for the pixel $t_{q r}$, (l) the magnitude and direction of total induced current density vector, $\vec{J}_{t_{r r}}=0$.

A single null pixel in a 13-coil array is depicted in Fig. 3(i) along with its simulated E-field in Fig. 3(j). An enlarged view of this array's central coil, in Fig. 3(k), reveals the requisite coil currents and phase vectors that induce the current density vectors to cancel or null the pixel $t_{q r}$. Using Eqn. (1), (2) and (3) yield the magnitude and direction of total induced current density vector, $\vec{J}_{t r}=0$ illustrated in Fig. 3(1) (note: unity is used for peak drive coil current magnitudes for simplification). 
(a)

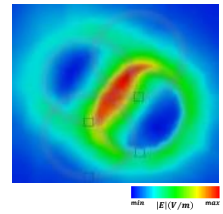

(b)

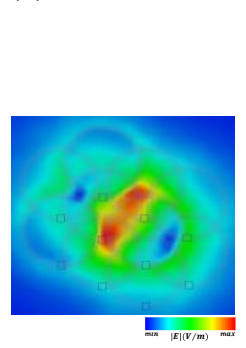

(c)

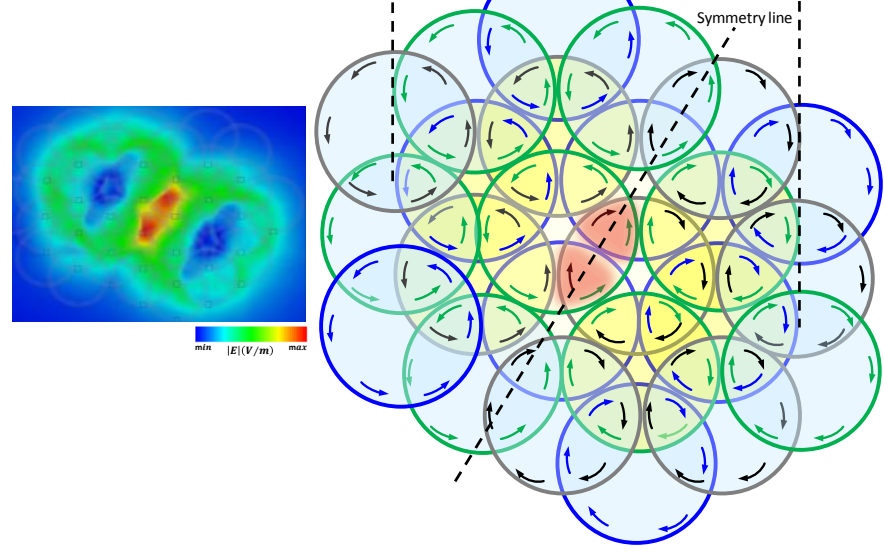

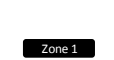
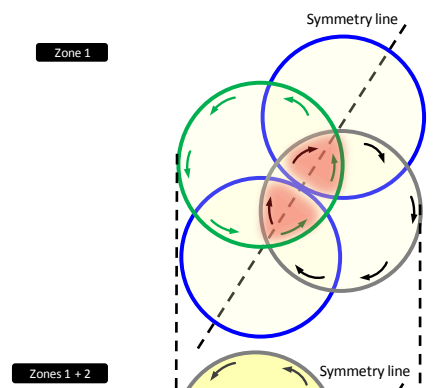

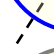

Fig. 4. The template array, with three variants or zones, is pre-loaded with coil current and phase parameters creating a double-pixel unit of construction for pattern formation. (a) zone 1 (4 coils), (b) zones $1+2$ (10 coils) and (c) zones $1+2+3$ (32 coils).

\section{Excitation Pattern Synthesis with the Template Array}

The next component in our synthesis method, and perhaps the most unique, is called the template array. Three variations or zones shown in Fig. 4, when pre-loaded with a set of coil parameters, create a desired pixel unit (e.g., double-pixel) in which the template array aligns and transfers this data to the recipient array for pattern synthesis. These steps repeat $n$ number of steps until pattern completion. The double-pixel, in Fig. 4 and 5, is uniquely suited for synthesis as the surrounding return currents, in all three layers of the array, are spread out and cancel as they move away from the excitation area. As such, this variant is used throughout this paper as the primary unit of construction.

As the zones are added there is also an increase in the magnitude of the induced E-field (approximately 10\%) due to the coincident current phases of the neighboring coils shown in Fig. 4(b) and (c). The increase in coils, as each zone is added, allows for additional paths to spread return currents farther out and away from the excitation pixel.

The next step in pattern synthesis is now described in Fig. 5 (a) whereby user-defined currents and phases in the template array are transferred to the underlying recipient array for the construction of a desired pattern or multiple patterns. The general concept is illustrated in Fig. 5(b) whereby the template array steps, repeats and transfers the identical coil parameter in the three designated positions. This is followed by the superposition of each the pixel current density array pattern from each step, seen in Fig. 5(b), giving rise to the unilinear pattern and the supporting simulations shown in Fig. 5(c).

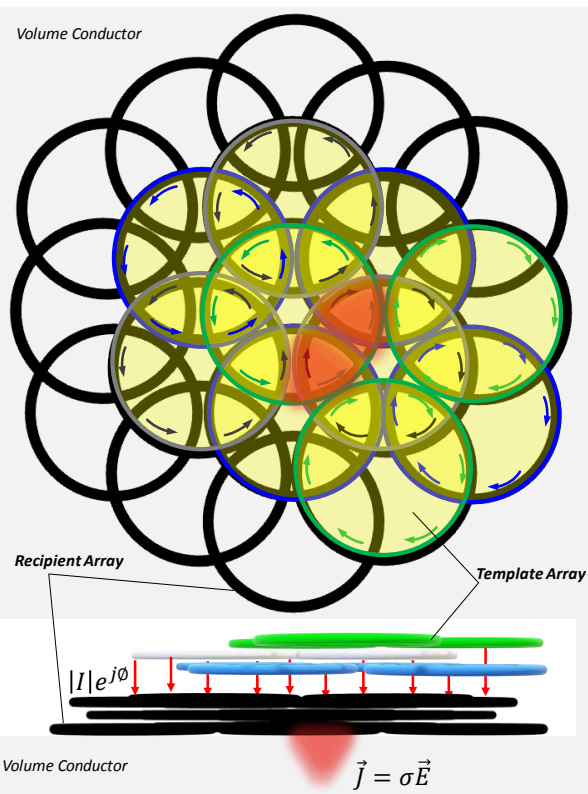

(a)
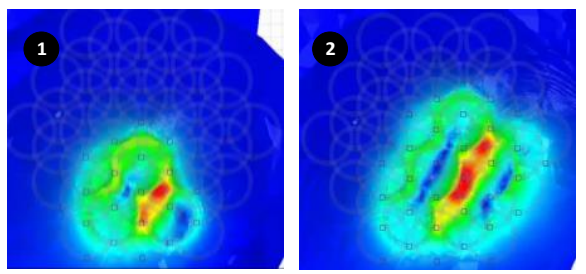

(c)

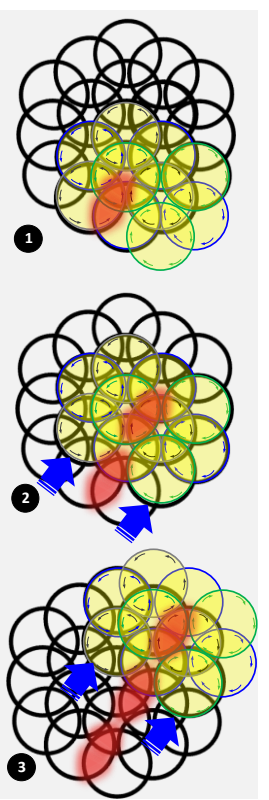

(b)

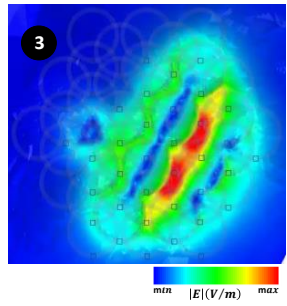

Fig. 5. Synthesis of a pattern with the template array using a double-pixel cell as the unit of pattern construction (note: excitation site in red). (a) pretranslation; a template array aligns with recipient array and transfers the userdefined current magnitudes and phases, (b) Post-translation; a series of steps 1 -3 are repeated to form a user-defined pattern, (c) FEM of the post-translation a series of steps $1-3$.

In each step, the template array is aligned on the hex grid via cubic coordinates above the recipient array (i.e., array used for excitation), as illustrated in Fig. 5(b). The template array's sole function is to act as a pixel "stamp" that imprints the pattern to the recipient array as shown Fig. 5(b). A supporting simulation is shown in Fig. 5(c). For a desired pattern then a step and repeat pattern would ensue accordingly until the pattern is complete. These pre-loaded parameters resident in the template array involve the user-defined fundamental unit of construction that is selected for pattern formation. Thus, given an a priori selected coil current magnitude and phase they are transferred 
resulting in a fundamental pixel unit. This induces E-fields and eddy currents $(\vec{J}=\sigma \vec{E})$ in a conductive medium for userdefined pattern information to the underlying recipient array.

\section{Cubic Coordinates and Axial Coordinates}

The synthesis component that facilitates the rotation and translation of coil parameters for synthesis is that of the cubic coordinate system which is mapped to a hexagonal grid. Cubic coordinates on a hexagonal grid allow for easy rotation and translation of pixel cells over that of square grids mapped to cartesian coordinate systems. This is due in part to the fact that they have only six equidistant neighbors as opposed to eight unequal distances in a square grid. As such, a simple system of coordinate transformation negates the need for trigonometric functions as seen in square and triangular grids. Also, axial coordinates, a close relation to cubic coordinates, allows for easy storage of coil current and phase parameters. After movement is completed, then cubic coordinates are converted to axial coordinates for data storage and source indexing for the coils in HFSS [16].

Several basic movements are described in Fig. 6 using cubic coordinates when mapped to a hexagonal grid. The center point of every coil in the dense array is referenced to the center hexagon (note: hexagons are color coded to match the colors of the coils in Fig. 2 and 6) and the associated cubic coordinate. The general constraint for cubic coordinates is

$$
x+y+z=0
$$

A clockwise $(\mathrm{CW})$ rotation around the grid, in Fig. 6, is achieved by a simple transformation of cubic coordinates every $60^{\circ}$ using

$$
(x, y, z) \rightarrow-(z, x, y)
$$

A counterclockwise $(\mathrm{CCW})$ rotation, in Fig. 6, around a specific ring is achieved by a simple transformation of cubic coordinates every $60^{\circ}$ using

$$
(x, y, z) \rightarrow-(y, z, x)
$$

Translation from hex flat-to-flat, seen in Fig. 6 (red arrows), for a given direction changing one of the 3 cube coordinates by +1 and changing another one by -1 (the sum must remain 0 ). For example, moving away $240^{\circ}$ on the hex grid between the $+y$ and $-z$, will result in adding 1 to $y$ and subtracting 1 from $z$.

$$
\begin{gathered}
0^{\circ} \Rightarrow(x, y, z) \rightarrow(x+1, y-1, k) \\
60^{\circ} \Rightarrow(x, y, z) \rightarrow(k, y-1, z+1)
\end{gathered}
$$

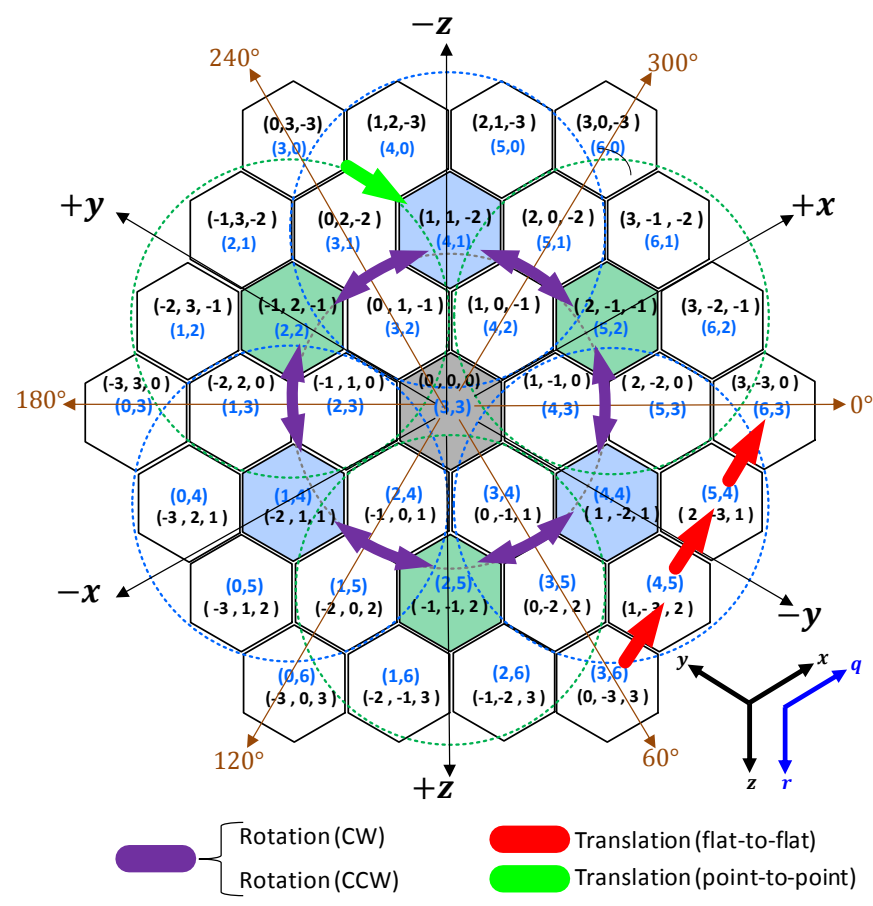

Fig. 6. Movement using cubic coordinates (note: in black font) on a hexagonal grid and axial coordinates (note: in blue font) are used for data storage (note: the axial coordinates are re-referenced for array storage).

$$
\begin{aligned}
& 120^{\circ} \Rightarrow(x, y, z) \rightarrow(x-1, k, z+1) \\
& 180^{\circ} \Rightarrow(x, y, z) \rightarrow(x-1, y+1, k) \\
& 240^{\circ} \Rightarrow(x, y, z) \rightarrow(k, y+1, z-1) \\
& 300^{\circ} \Rightarrow(x, y, z) \rightarrow(x+1, k, z-1)
\end{aligned}
$$

Translation diagonally, in Fig. 6 (green arrow) from hex vertex-to-vertex involves changing one of the three cubic coordinates by \pm 2 and the other two by $\mp 1$ with the sum remaining 0 per Eqn. (1).

Corresponding axial coordinates (blue font) are mapped in Fig. 6 as well. They are closely related to cubic coordinates using two of the three cubic coordinates. Whereby the $\mathrm{x}$ and $\mathrm{z}$ coordinates are re-assigned from $x \rightarrow q$ and $z \rightarrow r$ with y not required for this case. The general storage array for coil parameters after conversion from cubic to axial coordinates for the 7-coil array on a three-ring hex grid are shown in Fig. 6 and is of the order $(q \times r)=(6 \times 6)$. This general representation for storage array in Eqn. (10) shows that the nulls are used to fill in the unused sections of the pre-rotation and post-rotation values in the array due to the hexagonal shape of the grid (i.e., we employ a flat top hexagonal shaped array consisting of hexagons).

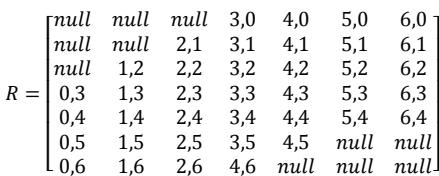




\section{Rotation and Translation of Pixels}

The fundamentals of rotation and translation of a pixel for pattern formation are illustrated in three examples. We start with the template array, in its smallest form consisting of four coils forming zone 1 . When the template array rotates it transfers pre-loaded coil current parameters by moving from coil hex-center to coil hex-center as shown in Fig. 7(a) - (b) where the center hexagon of each coil is highlighted by the corresponding color of the coil. An example of a simple counterclockwise rotation $(\mathrm{CCW})$ of a double pixel, along the $2^{\text {nd }}$ ring, using the zone 1 template array is shown in Fig. 7 illustrates the concept.

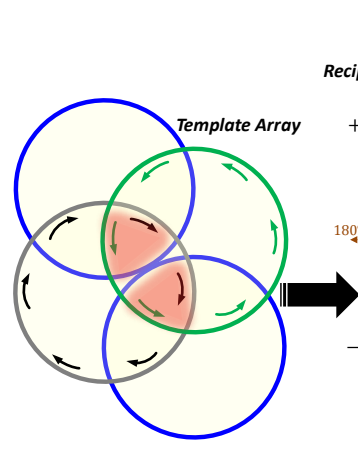

(a)

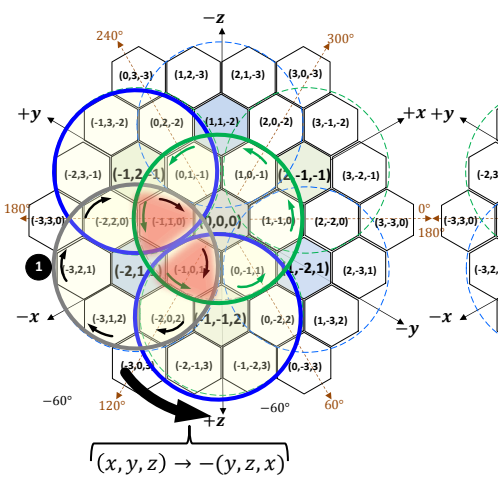

(c)

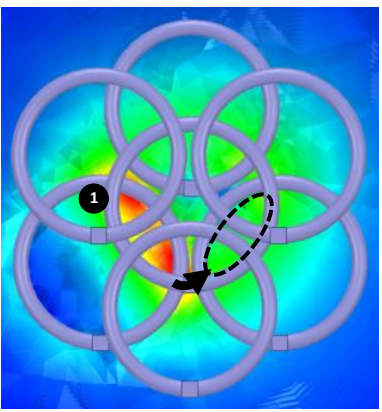

(e)

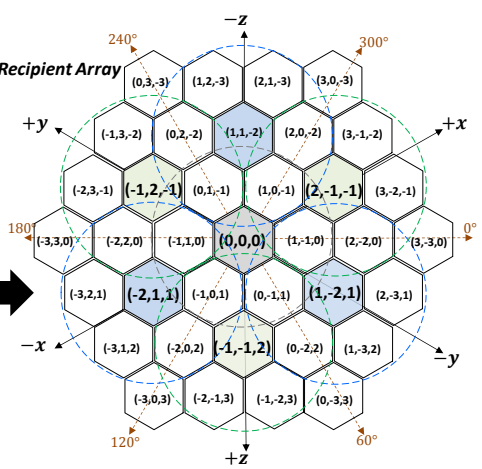

(b)

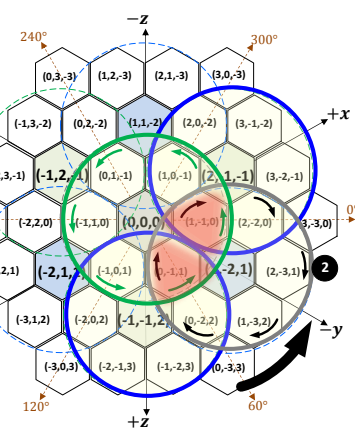

(d)

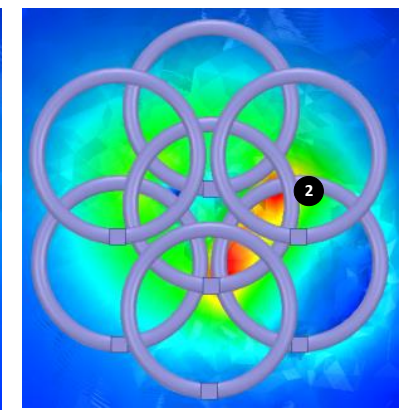

(f)

Fig. 7. Rotation $(\mathrm{CCW})$ of the pixel ( $2^{\text {nd }}$-ring) using the zone 1 template array. (a) template array moves, aligns and transfers pre-loaded user-defined data, (b) template array moves and aligns with a three-ring recipient array, (c) position 1 alignment is established and coil parameters are transferred, (d) two $60^{\circ}$ rotations (two hex positions) occur from position 1 to 2 followed by coil parameter transfer, two hex jumps by shifting coordinates and multiplying by 1, (e) E-field simulation of Position 1 and, (f) E-field simulation of Position 2.

Initially the template array, pre-loaded with coil parameters, moves and aligns with the recipient array to position 1 in Fig. $7(\mathrm{c})$ and the coil parameters are transferred. Then moving $\mathrm{CCW}$ two slots over which is equivalent to two $60^{\circ}$ rotations (two hex slots) to position 2 in Fig. 7(d) followed again by the transfer of coil parameters. This involves two hex jumps over and multiplying by -1 per Eqn. (3). E-field simulations in Fig. 7(e) - (f) show the movement of the double-pixel from position 1 of position 2. Using the same conditions but rotating clockwise (CW) Fig. 8 displays how the sparse arrays are populated with the parameters during each step along the $2^{\text {nd }}$ ring, of the template array.

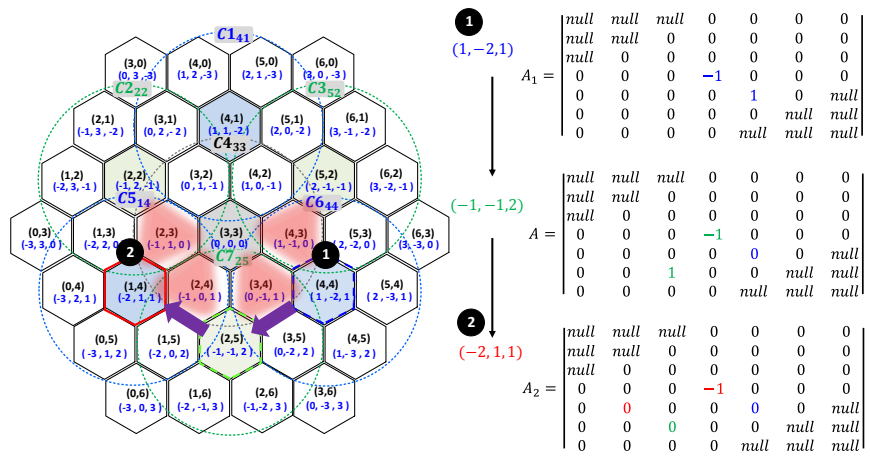

Fig. 8. Populated arrays for $\mathrm{CW}$ rotation with the zone 1 template array.

An example of a simple translation of a double pixel moving one hex slot along $0^{\circ}$ using a zone 1 template array is described in Fig. 9(a). In the same fashion, the template array with preloaded coil parameters, moves, aligns and transfers data to the recipient array from position 1 to position 2 .

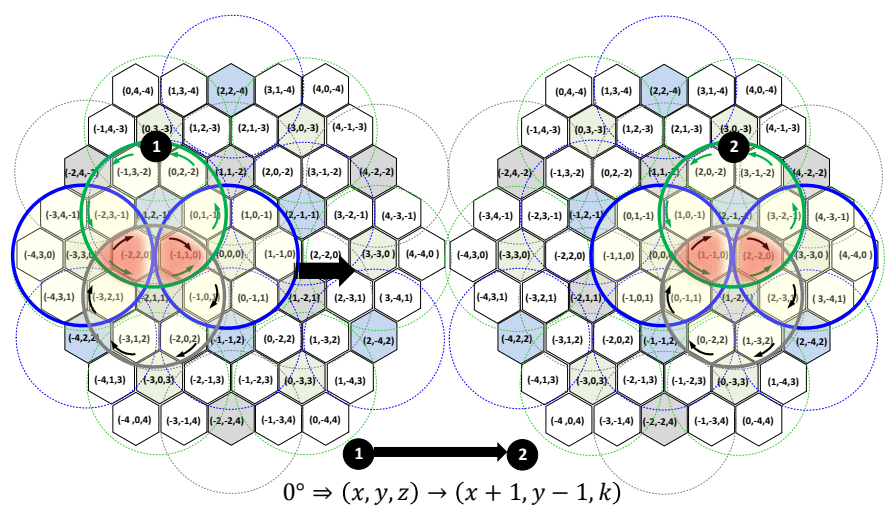

(a)

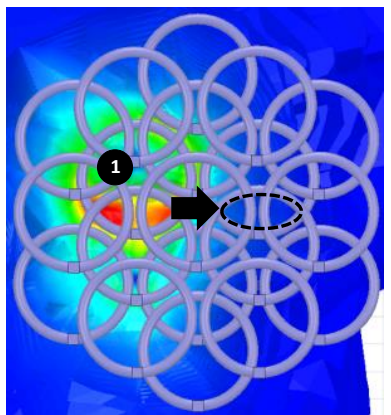

(b)

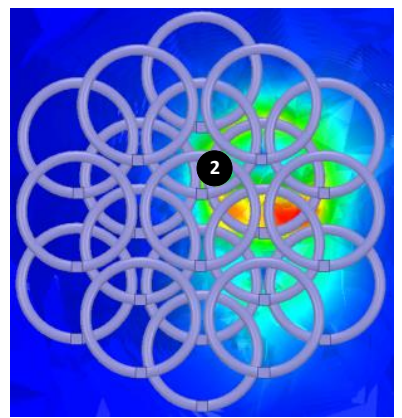

(c)

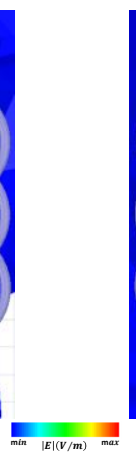

Fig. 9. Translation using the zone 1 template array across a 19 coil four-ring recipient array. (a) template array moves three-slots hex flat-to-flat at $0^{\circ}$ from position 1 to position 2, (b) E-field simulation of position 1 pre-translation pixel and, (c) E-field simulation of position 2 post-translation pixel. (note: $k=$ constant ). 
This is accomplished in the same way by moving from coil hex-center to coil hex-center as shown in Fig. 9(a). E-field simulations in Fig. 9(b) - (c) show the movement of the doublepixel from position 1 of position 2. To reiterate, the reference points being coil center to center is referenced to the center hex. The centers of each coil are highlighted by the corresponding color of the coil. In this case the translational movement is defined by Eqn. (4). E-field simulations in Fig. 9 (b) - (c) show the movement of the double-pixel from position 1 of position 2 .

A final example using a larger template array (zone $1+2$ with 10 coils) is shown in Fig. 10. A rotation $(\mathrm{CW})$ of $60^{\circ}$ along the hex centers of the $2^{\text {nd }}, 3^{\text {rd }}$ and $4^{\text {th }}$ rings with the template array over an 8-ring recipient array is shown in Fig. 10(a). The rotational (CW) step of $60^{\circ}$ shift of coordinates using Eqn. (2). In Fig. 10 (a) the template array rotates $60^{\circ}$ from position 1 to position 2, with supporting simulations shown in Fig. 10 (b) (c) on the movement of the excitation double-pixel. The simulation in Fig. 10 (d) shows the superposition of currents in the two sets of template array parameters which reveal the emergence of a curved pattern seen in Fig. 10 (d).

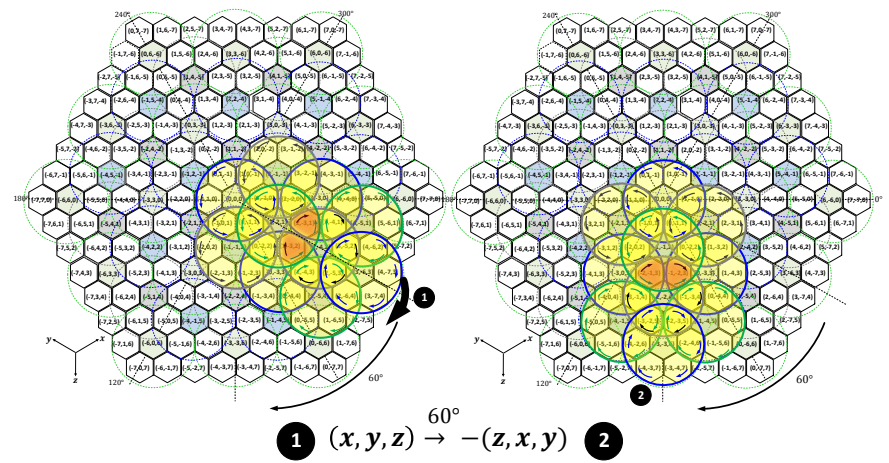

(a)

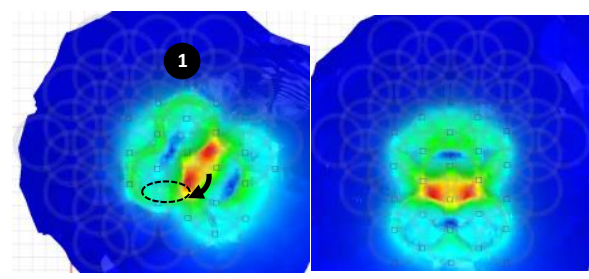

(b)

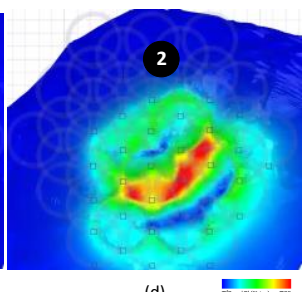

Fig. 10. Rotation $(\mathrm{CW})$ of $60^{\circ} 2^{\text {nd }}, 3^{\text {rd }}$ and $4^{\text {th }}$ rings with the template array (zone 1 and 2 consisting of 10 coils) over an 8-ring recipient array. (a) template array rotates $60^{\circ}$ from position 1 to position 2 ; rotation $(\mathrm{CW})$ of $60^{\circ}$ via the shift of cubic coordinates and multiplying by -1 , (b) simulation of position 1 of excitation pixel (excitation area in red), (c) simulation of position 2 of excitation pixel, (d) simulation shows the superposition of currents via addition of two arrays yielding a curved pattern.

Code using the Python mathematical library NumPy [14] was written to move and transfer coil parameters around the grid via the template array while indexing all three currents (magnitude and phase) in all three coil layers. A top-level flowchart, in Fig. 11 , outlines each major step in the code.

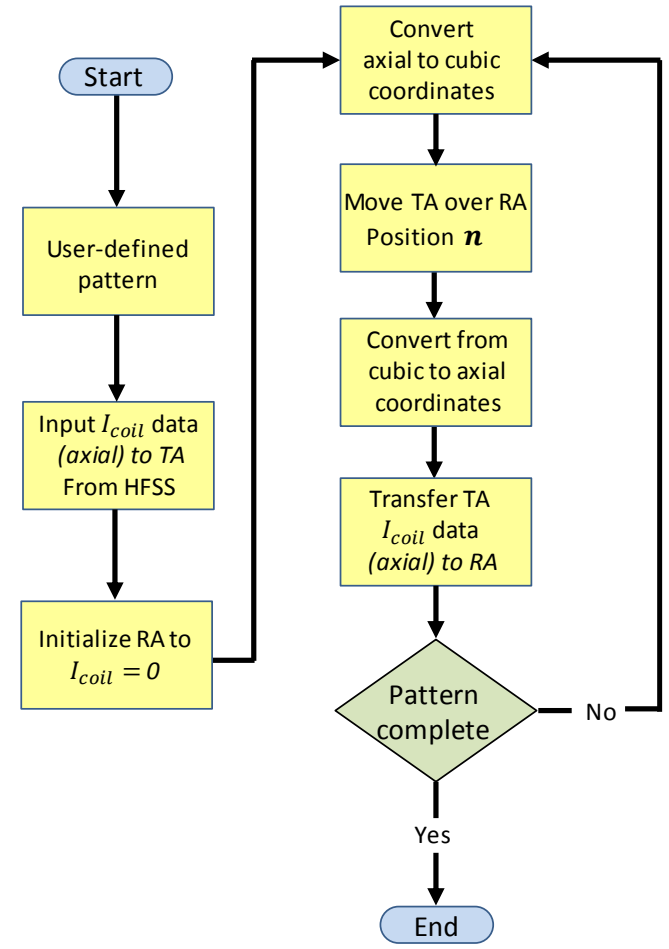

Fig. 11. Top level pattern synthesis flow chart with using NumPy [14] the mathematical library in Python.

\section{METHODS AND APPARATUS}

\section{E-field Measurements}

The amplitude of the induced E-field depends on magnetic field amplitude, how fast it changes over time and the direction. In terms of spatial distribution, the magnetic field that is generated by the current in the stimulation coil is almost independent from the specific head anatomy, whereas the induced E-fields in the biological media are not [17],[18]. As such, we developed a rotating dual dipole probe in Fig. 12(b) [19],[20] that when immersed in polyvinylpyrrolidone solution (PVP) [21], a cortical equivalent solution, can measure the potential difference between the two probes tips. A tissueequivalent solution of PVP solution $(16 \mathrm{mS} / \mathrm{cm})$ [21]-[22] was used for all E-field measurements. For the basis of equal comparison each individual coil was energized and monitored to have identical voltages and currents (24V and $8 \mathrm{~A}$ ) throughout each measurement session.

A low noise differential amplifier [23], in Fig. 12(a) was used to amplify and filter the resulting voltage waveform. Measured data was recorded on an oscilloscope and stored in memory for post-processing using Matlab [24]. The relationship for the measured voltage is

$$
V_{\text {meas }}=\Delta V / G
$$

where $\Delta V$ is the potential difference at the dipole tips and $G$ is the gain of differential amplifier. 


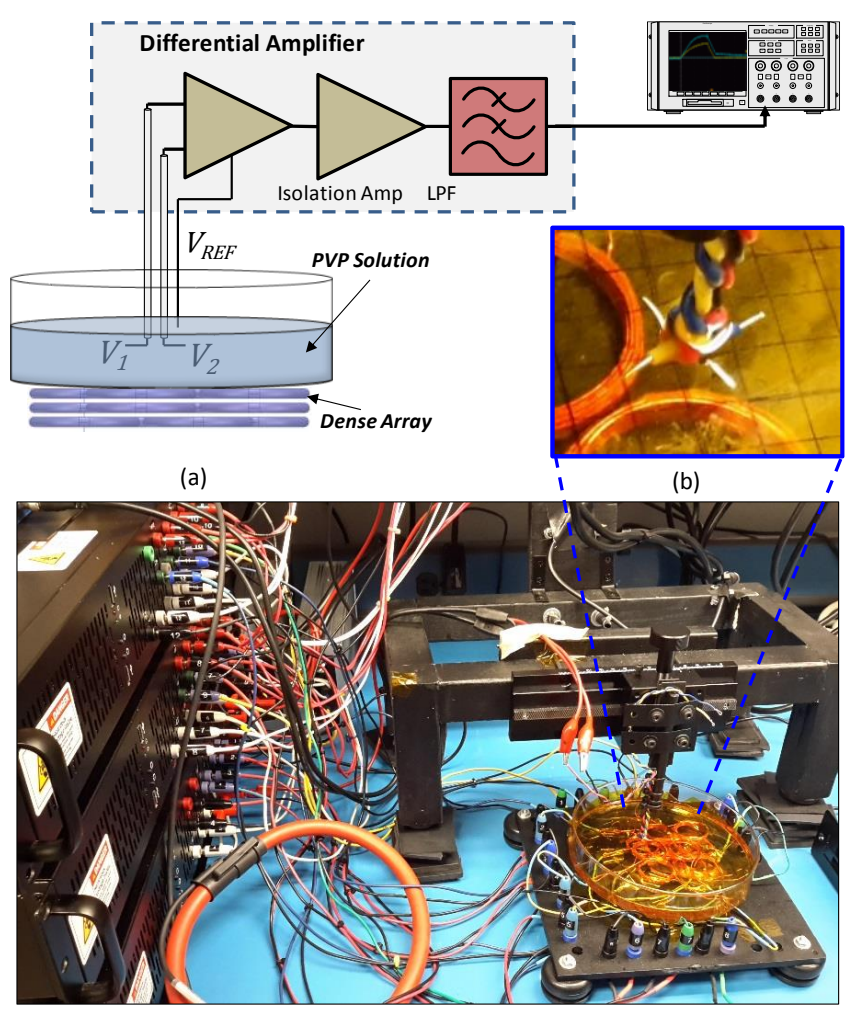

(c)

Fig 12. E-field and eddy-current measurement in PVP solution. (a) block diagram of measurement method, (b) photograph of dual dipole rotary probe immersed in PVP solution over the dense array, and (c) photograph of the system, and 13-coil dense array under test in PVP solution [21].

A precision $\mathrm{z}$-axis translation stage [25] with $1.0 \mathrm{~mm}$ steps mounted on a custom stabilization fixture in Fig. 12 (c) was fabricated and used for precision profile measurements. A Rogowski coil [26] shown in the Fig. 12 (c) photograph, was used to measure the time-varying drive current delivered to each coil in the array.

\section{SYSTEM ARCHITECTURE}

The scalable modular architecture of our system, is shown in Fig. 13, consisting of multiple $3 \mathrm{U}$ chassis that pulse current to various test array topologies with either monophasic or biphasic waveforms. The main components shown in the system block diagram are the dual insulated-gate bipolar transistor (IGBT) driver/switch/energy storage assemblies in each $3 \mathrm{U}$ chassis demonstrating that the IGBTs and drivers in the system which are rated for higher voltages and currents of $1200 V_{\text {peak }}$ and 600 $I_{\text {peak }}$ respectively. Increasing energy storage capacity and putting the IGBTs in parallel can accommodate twice the current carrying capability delivered to the dense array. One dual gate driver per dual IGBT per channel switches the selected current (positive or negative) to each coil in the array. Independent control of all channels (positive or negative) is in two programmable clock driver circuits $(1: 10)$, feeding each dual gate drive. The clock driver is synchronized to a common clock resident in the arbitrary waveform generator (AWG). A more detailed treatment of the electronics and system architecture was published earlier [27].

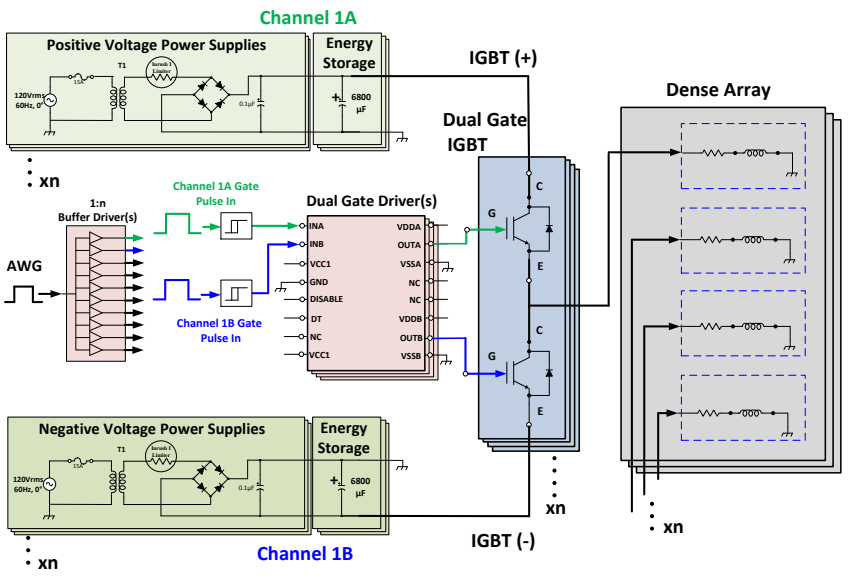

Fig. 13. Multichannel Exciter and Dense Array System Block Diagram.

\section{EXPERIMENTAL RESULTS AND SIMULATIONS}

\section{Traditional Patterns}

The multifunction capabilities of our array are first demonstrated in Fig. 14 by simulating the patterns of the traditional single function systems using figure-8 coil topologies Fig. 14 (a). The E-field measurements shown in Fig. 14 (b)-(c) are in good agreement with the patterns seen in the literature [28],[29]. This also baselines and validates our measurement approach.
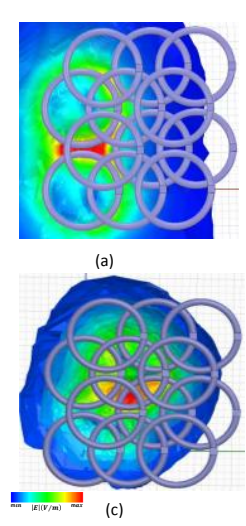

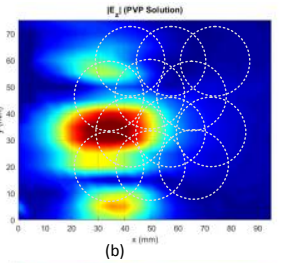

(b)

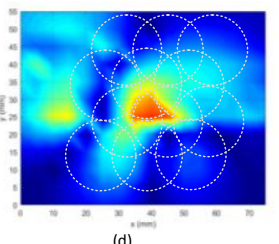

(d)

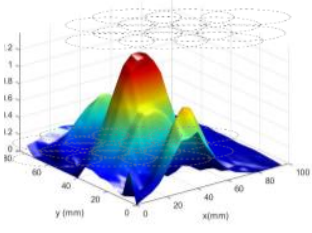

(c)

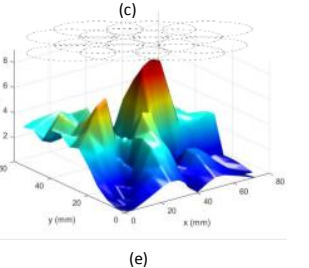

Fig. 14. Comparison of the E-field measurements vs. simulations of a figure- 8 and a single pixel both generated in a 12-coil dense array. (a) simulation of figure-8, (b) - (c) measurement of figure- 8 E-field pattern validating the versatility of array by forming a traditional pattern, $(d)-(e)$ measurement of a single pixel E-field pattern.

A radial E-field scan measurement of a selected pixel, in a 12-coil array, is compared with two different figure-8 topologies under the identical conditions in Fig. 15. Notably the E-field focal areas are very similar. One excitation lobe of the pixel is slightly elevated on the left side and lower on the right side since the entire array is energized in this specific case. As will be shown later in the paper, adjusting charging voltages on each relevant coil, can be employed for optimization of various patterns. 


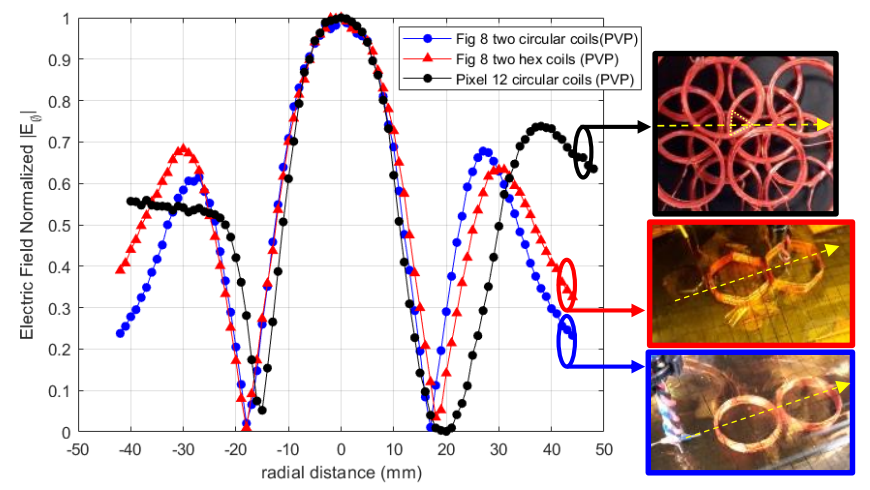

Fig. 15. Radial near field scan of the E-field comparing three different coil topologies; a figure- 8 with circular coils, a figure- 8 with hexagonal coils and a pixel in a 12-coil array (note: yellow line shows trajectory of near field probe).

\section{Complex Use-Defined Pattern Formation}

The methods, previously discussed, by which the template array travels over the recipient array was first verified using a light table with transparencies and then assessed in HFSS. This laid the foundation for the generation of Python (NumPy) code [14] to generate the various functions for coordinate conversion, movement, data storage and excitation source profiles that could be directly ported in to Ansys HFSS [16]. The template array providing each coil's parameters (magnitude and phase) to the recipient array in HFSS allows for a review and check of the excitation before proceeding. Since this is a post simulation process function, only one analysis is required in HFSS. Therefore, each appended data set can be uploaded into the source excitation profile in HFSS providing a timely update of E-field and current density vector contours as the pattern is created.

A series of simulations and experimental results are now discussed with the goal of validating the procedure described earlier. We begin by synthesizing a circular pattern in Fig. 16. using a template array (zone $1+2)$ transferring data to a 19coil recipient array. This circular pattern offers the capability of electronically changing the effective diameter of a coil and subsequent excitation pattern which could potentially offer rapid switching of spatiotemporal reconfigurable depths of penetration. Notably the capability of electronically changing the diameter of a circular pattern in Fig. 16 shows that the reconfigurable diameter of a circular pattern can be increased for deeper depth of penetration in a given media. Thus, offering a unique variable that allows the user to rapidly reconfigure depth of penetration for spatiotemporal optimization and perhaps alternate the modality with others at different sites and depths with fast switching. This modality would be very hard to implement in today's single function systems. Detailed measurements reporting on this technique are described in our previous paper [27].
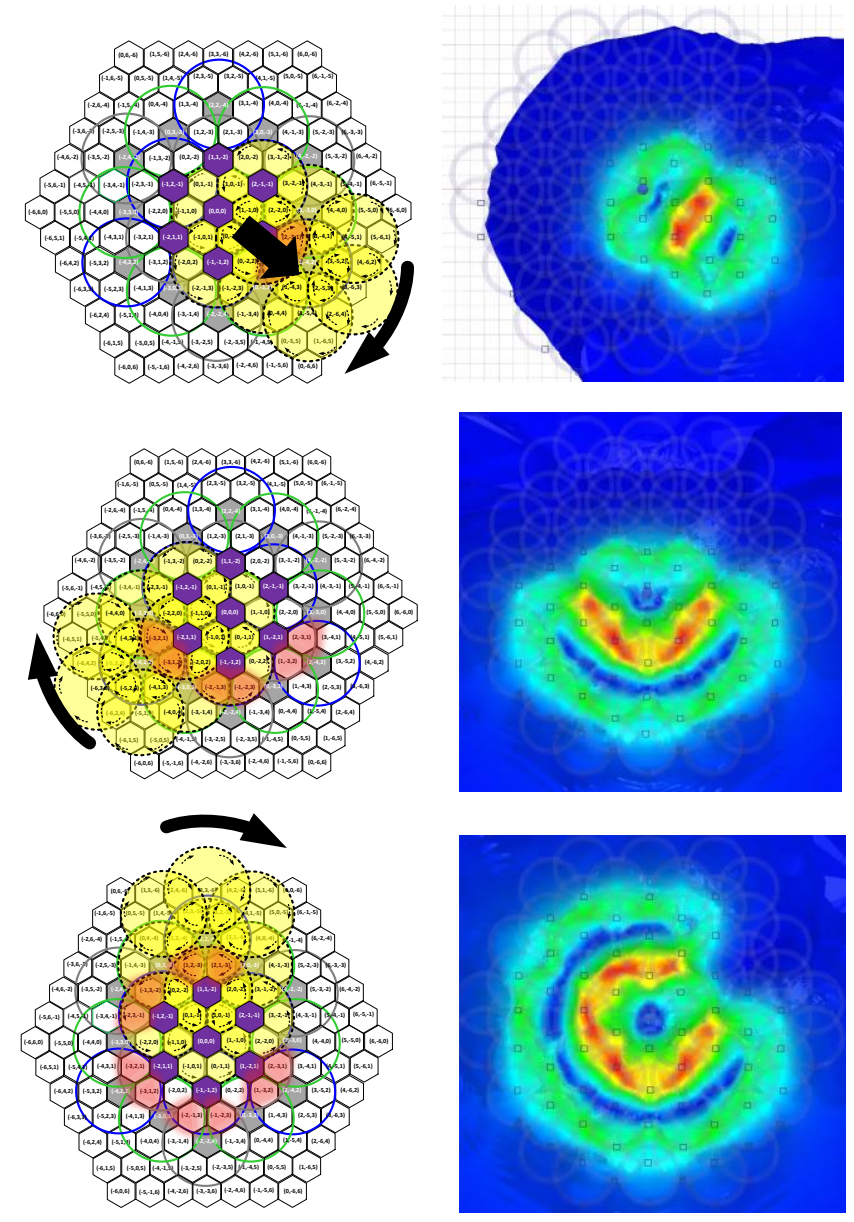

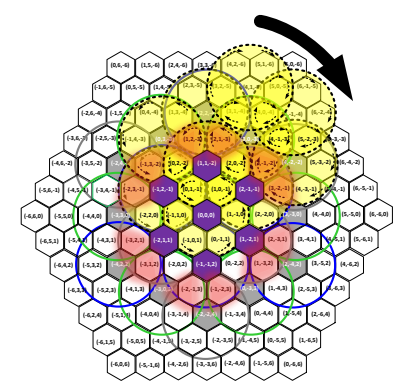

(a)

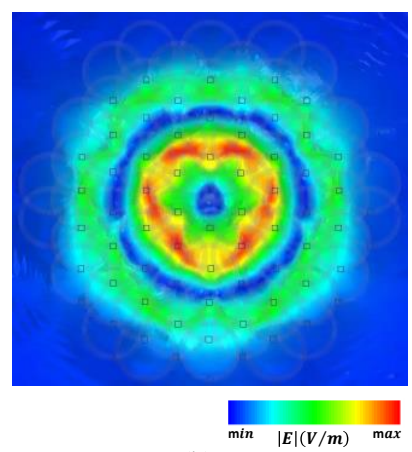

(b)
Fig. 16. Movement of a double-pixel (one translation step and multiple rotations) to form a reconfigurable circular pattern. (a) illustration of the sequence of pixels generated, rotated and transferred by the template array to the recipient array to construct a circular pattern, (b) simulation of the sequence.

Another interesting capability, demonstrated in Fig. 17, is the formation of complex user-defined patterns (e.g., zig - zag). This potentially could be of great utility. For example, when coupled with neuro-navigation it could provide geometrically optimal angles of excitation trajectory (e.g., orthogonal to a sulci [30]) in the brain as it pertains to the unique characteristics of each patient. 

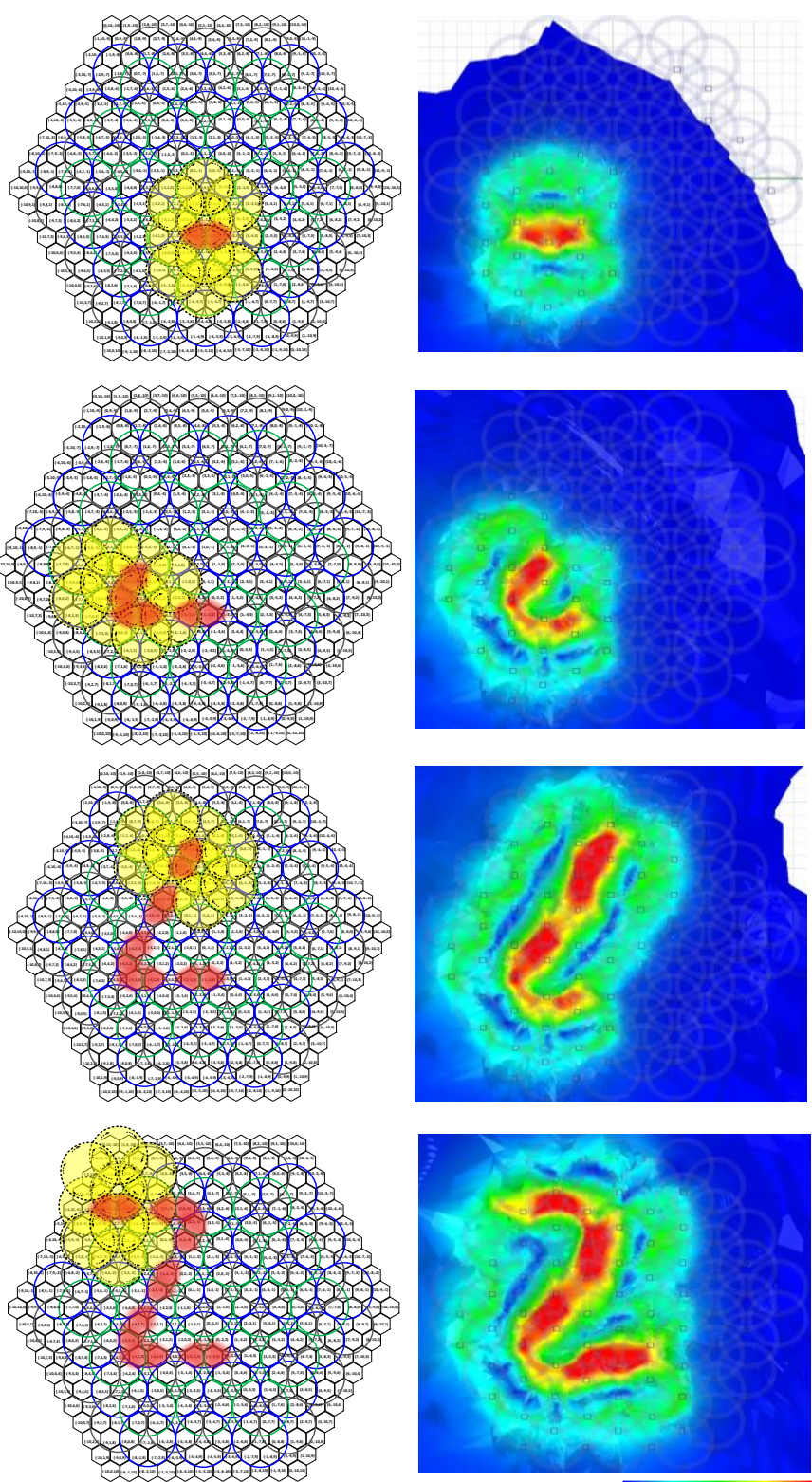

(a)

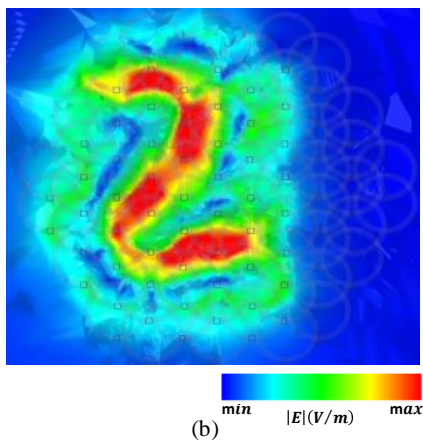

Fig. 17. Movement of a double-pixel (one translation step and multiple rotations) to form a zig-zag pattern. (a) illustration of the sequence of pixels generated, rotated and transferred by the template array to the recipient array and, (b) simulation of the sequence.

A series of measurements vs. simulations for five different rudimentary patterns are presented and discussed in Fig 18. The goal being to validate the procedure experimentally. Measured vs. simulated for all results in Fig. 18 show good agreement. We begin by measuring a single double-pixel pattern in Fig. 18 (a) using a zone 1 template array followed by an "omega" shaped pattern in Fig. 18 (b) using three double-pixels. A multisite pattern follows with each excitation consisting of a double - pixel in a 12-coil array. Using two-pixels per pattern and a zone $1+2$ template array a unilinear and a curved pattern formed by two pixels in Fig. 18 (d) and (e).

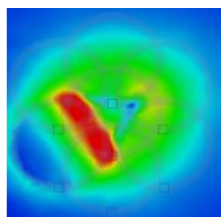

(a)

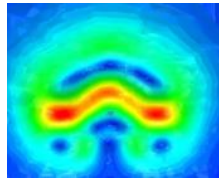

(d)

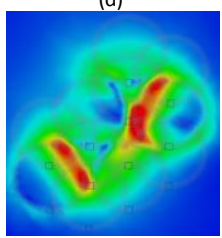

(g)
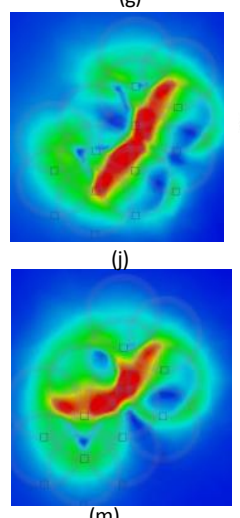

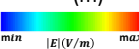

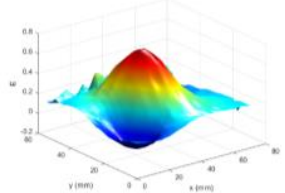

(b)

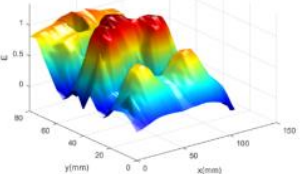

(e)

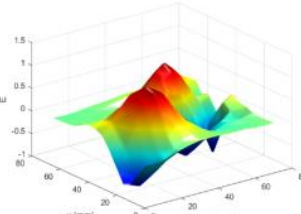

(h)

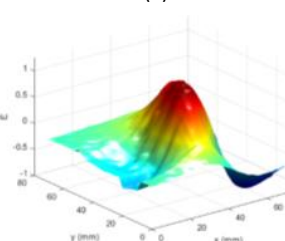

(k)

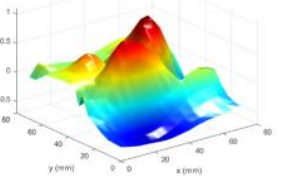

(n)

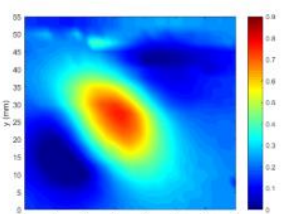

(c)

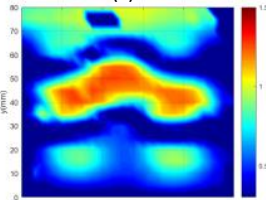

(f)

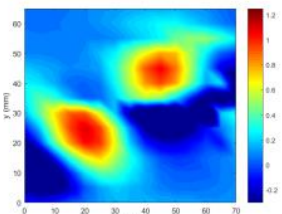

(i)

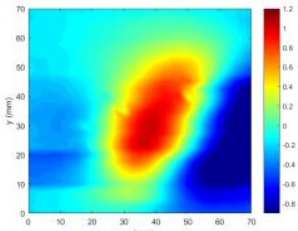

(I)

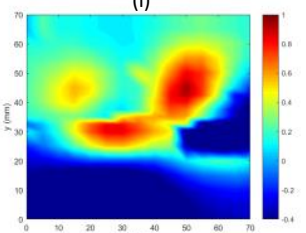

(o)
Fig. 18. Simulations (left side) vs. measurements (center and right side) of arbitrary E-field patterns. (a)-(c) single double-pixel in 7-coil array, (d)-(f) "omega" pattern in a 12 coil array using three single double pixels (g)-(i) two multi-site in a 12 coil array (two double-pixels), (j)-(l) unilinear pattern diagonal pattern in a 12-coil array and (m)-(o) curved pattern; superposition of two double-pixels in a 12-coil array.

\section{Proximity Effects}

As coils are increased in multichannel arrays proximity effects due to mutual coupling, degraded $\mathrm{Q}$ and distorted current waveforms caused by unwanted coil to coil interaction will be a significant design challenge. However, we propose that this effect can be mitigated by our ability to power down, ground non-energized coils and control individual coil current levels by using programmable charging voltages as was the investigated in an earlier paper by Han et al [31]. Moreover, the addition of a n-port switch matrix, although not developed for this current study, would significantly limit the number of coils that are energized at a time and would also provide another degree of freedom for optimization of power efficiency and thermal management. Therefore, when a large coil array is used only a small fraction of the coils are used to generate the patterns demonstrated in this study.

The mitigation techniques we propose are reasonable but clearly numerous experiments at higher current levels will be 
required to better understand this design challenge brought on by the highly populated architecture of a three-layer array of coils. Nonetheless not the focus of this paper, this challenge, regardless of coil diameter, can degrade current waveforms so it is prudent to address this albeit briefly in this study. To that end preliminary measurements of the voltage waveforms as affected by coil position was undertaken.

A worst-case condition and an ideal control case were established to evaluate coil-to-coil interaction effects in the 12coil dense array shown in Fig. 19. The worst-case waveform degradation occurs when the two coils are vertically aligned center to center along the z-axis as in Fig. 19 (blue coils). The ideal or isolated single coil case (i.e., absence of coupling) is shown in Fig. 19 (red coil). A nominal coil position in the array was selected with the overlap condition shown in Fig. 19 (green coils) for coils 1 and 5 in the array. The charging voltages were initially held constant for these two coils which resulted in a decrease in the voltage waveform in Fig. 19 (green). This was then compensated for and brought back to the isolated case voltage level in Fig. 19 (black). Thus, the mitigation of waveform degradation is feasible by adjusting the voltage on the charging capacitors for each channel. However, more research remains as this is one case on the outside of the array. Future implementations using programmable power supplies controlled by a master system processor could be implemented to address this issue.

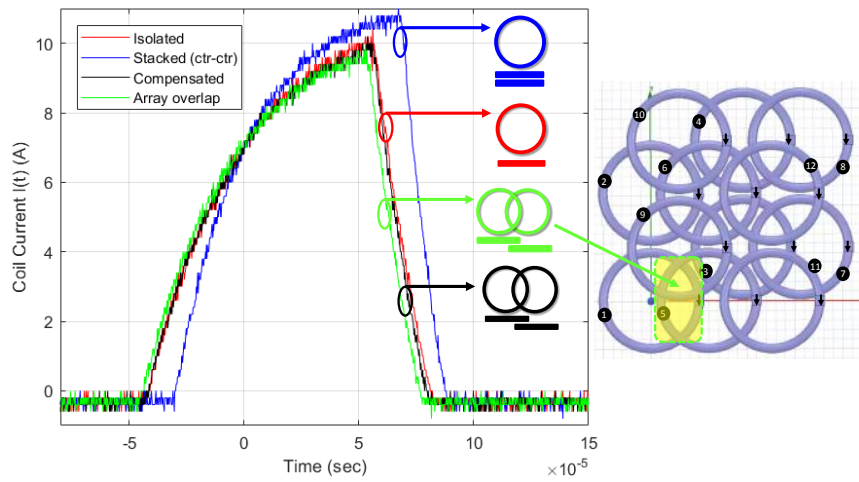

Fig. 19. Proximity effect mitigated by the adjustment of charging voltages.

The summary of patterns, in Fig. 20, demonstrates the broad categories of user-defined patterns that can be synthesized using the techniques described in this paper. The multi-locus (reversable current directions) and multi-site pattern in Fig. 20(a) has the potential to assist the researcher in brain connectivity studies which to date have been hampered by slow manual movement of one or two heavy stimulator coils [12]. Fig. 20(b) shows the arbitrary nature of a zig-zag pattern indicating that quite a large number of arbitrary geometrically optimal angles of trajectory of excitation across a sulci boundary at the appropriate angle are possible [30]. The circular pattern shows that the reconfigurable diameter of a circular pattern, examined in our previous work [27], can be increased for deeper depth of penetration in cortical tissue or modulated rapidly to alternate depths or sites. Sharp bends of the pattern, Fig. 20 (d) can be accommodated to adjust to the complex trajectories across a specific boundary of sulci in the brain unique to each patient. Unilinear patterns with the ability form at any angle are shown in Fig. 20 (e) - (f). In all these cases, the induced E-fields and current vectors of the patterns can be rapidly modified, reshaped and redirected with any number of spatiotemporal variations.

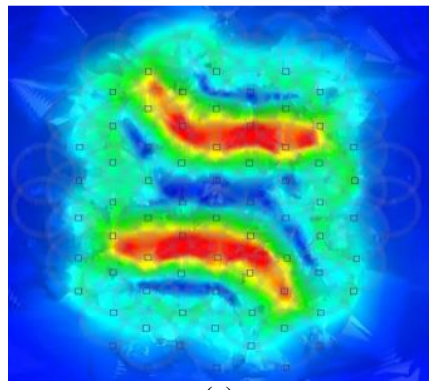

(a)

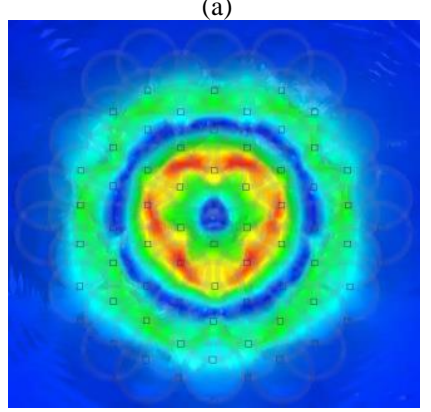

(c)

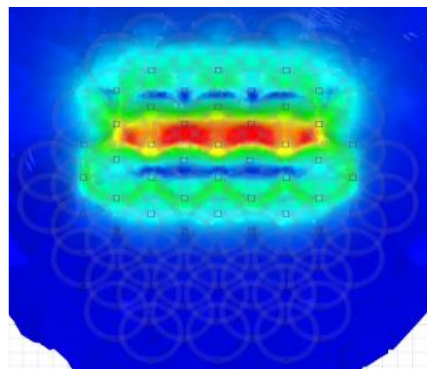

(e)

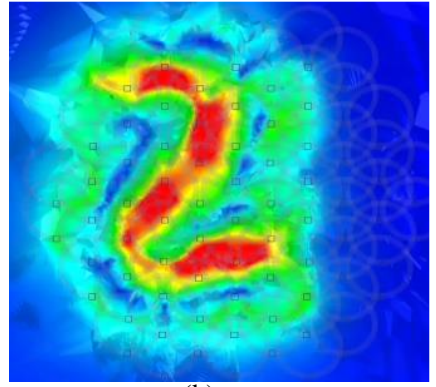

(b)

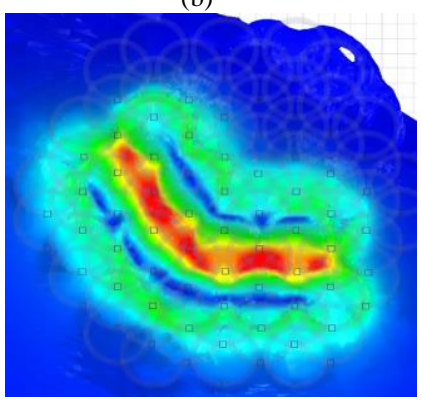

(d)

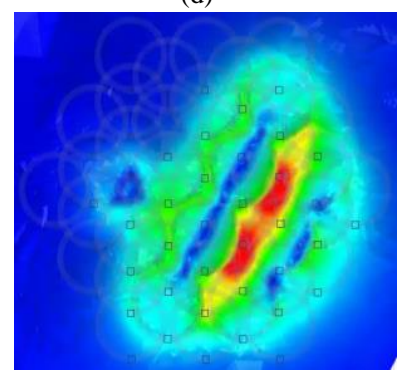

(f)
Fig. 20. Summary of user-defined synthesized patterns. (a) multi-site/multiloci, (b) zig - zag pattern, (c) circular for reconfigurable depth of penetration, (d) $45^{\circ}$ bend, (e) horizontal unilinear and (f) diagonal unilinear.

\section{CONCLUSION}

We report a practical synthesis method, as demonstrated by simulations and measurements, that can generate complex multi-locus and multi-site current patterning in a conductive medium for noninvasive neuromodulation and related biomagnetic applications. This method was enabled by our scalable dense magnetic coil array system first introduced in our earlier work [27]. The specific arrangement of the system's three mutually overlapping triangular coil arrays provide a mathematically complete set of excitation functions, with no dead zones, allowing any spatial current distribution to be created.

Our synthesis process successfully addressed the challenge of incorporating all three coils currents and array layers in an index system that rotates and translates easily. The index system selected used cubic coordinates for movement and axial 
coordinates for data storage. The two closely related coordinate systems were mapped to a hexagonal grid and NumPy [14], the mathematical library within Python 3, was used for the code. Python also proved to be a convenient interface in to HFSS [16] for the export and import of source definitions of the coil parameters as well. Excitation patterns were then created by a practical step and repeat pattern (e.g., double-pixel unit) preloaded in the template array that acted as a "transfer" vehicle to the recipient array.

In contrast to other existing approaches, the proposed array demonstrates the ability to control the direction of the induced eddy currents at multiple sites (sequential or simultaneous) which may prove useful for future treatments that could take advantage of this additional degree of freedom. For example, these modalities could induce current patterns either in a longitudinal or transverse trajectory to specific brain structures which may vary from patient to patient. In addition, this highlights the advantage of our approach in that it provides complete control over the current profile on the entire surface of the brain, allowing us to manipulate the spatial extent of the return currents. Through the independent control of currents, the redirection and dispersal of extraneous return currents and their activation levels can be minimized. This potentially could aid in improved precision and isolation in and around a target area especially when trying to improve isolation between multisites for brain connectivity studies. The capability of reduced extraneous excitation is the subject of the next paper by this team along with a more in-depth look at evaluating proximity effects due to coil to coil interactions. In addition, the potential benefits offered by integrating a switch matrix at the output of our multichannel exciter before being ported into the dense array will be explored.

\section{REFERENCES}

[1] C. P. Ashdown et al, S. C. Johns, "Pulsed Low-Frequency Magnetic Fields Induce Tumor Membrane Disruption and Altered Cell Viability," Biophysical Journal, 118, pp. 1552-1563, April 2020.

[2] Y. Liu, D. Chen, P. Shang and D. Yin, "A Review of Magnet Systems for Targeted Drug Delivery," Journal of Controlled Release, 302, pp. 90-104, 2019.

[3] L. M. Koponen, J. O. Nieminen and R. J. Ilmoniemi, "Multi-locus transcranial magnetic stimulation - theory and implementation," Brain Stimulation, vol. 11(4), pp. 849-855, April 2005.

[4] L. I. Navarro de Lara, M. Daneshzand, A. Mascarenas, D. Paulson, K. Pratt, Y. Okada, T. Raij, S. N. Markarov and A. Nummenmaa, " A 3axis coil design for Multichannel TMS Arrays," Neurolmage, 224, 2021.

[5] L. Hernandez-Garcia, T. Hall, L. Gomez and E. Michielssen, "A Numerically Optimized Active Shield for Improved Transcranial Magnetic Stimulation," Brain Stimulation, 3, pp. 218-225, 2010.

[6] E. W. Weisstein, "Reuleaux Triangle," MathWorld--A Wolfram Web Resource, https://mathworld.wolfram.com/ReuleauxTriangle.html.

[7] F. S. Salinas et al. "Detailed 3D Models of the induced electric field of transcranial stimulation coils," Physics in Medicine and Biology, vol. 52, pp. 2879-2892, 2007.

[8] A. Barker, R. Jalinous, and I. Freeston, "Non-invasive magnetic stimulation of human motor cortex," Lancet, 325, pp. 1106-1107, 1985.

[9] S. Ueno, T. Tashiro and K Harada, "Localized stimulation of neural tissues in the brain by means of a paired configuration of time-varying magnetic fields," Journal of Appl. Phys. vol. 64, pp. 5862-5864, 1988.

[10] A. Zangen, Y. Roth, B. Voller and M. Hallet, "Transcranial magnetic stimulation of deep brain regions: evidence for efficacy of the H-Coil," Clinical Neurophysiology, vol. 116(4), pp. 775-779, April 2005.
[11] L. J. Crowther, P. Marketos, P. I. Williams, Y. Meklikov, and D. C. Jiles, and J. H. Starsewski. (2011, April) Transcranial Magnetic Stimulation: improved coil design for deep brain investigation. [online]. Journal of Applied Physics, 109, Available: https://aip.scitation.org/doi/abs/10.1063/1.3563076.

[12] A. Guiffre, C. Kahl, E. Zewdie, "Reliability of Robotic transcranial magnetic stimulation," Journal of Neurophysiology, Vol 125, 1, Jan 202.

[13] J. Ruohonen and R. Ilmoniemi, "Focusing and Targeting of Magnetic Brain Stimulation using Multiple Coils," Medical and Biological Engineering and Computing", vol. 36, issue 3, pp. 297- 301, May 1998.

[14] Harris, C.R., Millman, K.J., van der Walt, S.J. et al. "Array programming with NumPy, "Nature 585, 357-362, 2020.

[15] A. Patel, Hexagonal Grids from Reed Blob Games, https://www.redblobgames.com/grids/hexagons/, May 2020.

[16] Ansys, Inc., High Frequency Structure Simulator (HFSS 2021 R1), Available: (https://www.ansys.com/about-ansys).

[17] S. Grehl, D. Martina, C. Goenvalle, Z. Deng, J. Rodger and R. Sherrard (2016, Nov.), In-Vitro Magnetic Stimulation: A Simple Stimulation Device to Deliver Defined Low Intensity Electromagnetic Fields. [online] Frontiers in Neural Circuits, available:https://www.frontiersin.org/articles/10.3389/fncir.2016.00085/ full.

[18] K. Esselle and M. Stuchly, "Neural Stimulation with Magnetic Fields: Analysis of Induced Electric Fields," IEEE Transactions on Biomedical Engineering, vol 39(7), pp. 693-700, July 1992.

[19] P. M. Glover, and R. Bowtell, "Measurement of Electric Fields due to Time-varying Magnetic field Gradients using Dipole Probes," Phys. Med. Biol., 52, pp. 5119-5130, 2007.

[20] F. X. Hart and K. W. Wood, "Eddy Current Distributions: Their Calculations with a spreadsheet and their measurements with a dual dipole antenna probe," Am. J. Physics, pp. 461-467, 59(5), May 1991.

[21] C. Ianniello, J. A. D. Zwart, Q. Duan, C.M. Deniz, L. Alon, J.-S. Lee, R. Lattanzi, and R. Brown, "Synthesized Tissue-Equivalent Dielectric Phantoms Using Salt and Polyvinylpyrrolidone Solutions," Magnetic Resonance in Medicine, vol. 80, pp. 413-419, 2018.

[22] "IEEE Recommended Practice for Determining the Peak Spatial-Average Specific Absorption Rate (SAR) in the Human Head from Wireless Communications Devices: Measurement Techniques," IEEE Std 15282013, September 2013, pp.1-246.

[23] AlphaLab Inc., Oscilloscope preamplifier LNA10, (www.alphalabinc).

[24] Mathworks, Matlab 19a, (www.mathworks.com).

[25] Edmund Optics, $125 \mathrm{~mm}$ travel translation stage (www.edmundoptics.com).

[26] Fluke Corporation, i2000 AC flex current clamp (Rogowski coil) (https://www.fluke.com).

[27] M. C. Smith, A. Li and D. F. Sievenpiper, "A Multifunction Dense Array System with Reconfigurable Depth of Penetration," IEEE Journal of Electromagnetics, RF and Microwaves in Medicine and Biology, vol. 5, no. 1, pp. 35-45, March 2021.

[28] Brain Stimulation Methodologies and Interventions. Irving M. Reti Editor. Hoboken, New Jersey: John Wiley \& Sons, Inc; 2015.

[29] Z. D. Deng, S. D. Lisanby, and A. V. Peterchev, "Electric field depthfocality tradeoff in transcranial magnetic stimulation: simulation comparison of 50 coil designs." Brain Stimulation, 2013, vol. 6 pp. 1-13.

[30] A. M. Janssen, T. F. Oostendorp, and D. F. Stegeman, "The coil orientation dependency of the electric field induced by TMS for M1 and other brain areas," J NeuroEngineering Rehabil, 12, 47, 2015.

[31] B. Han, I. K. Chun, S. C. Lee and S. Y. Lee, "Multichannel Magnetic Stimulation System Design Considering Mutual Couplings Among the Stimulation Coils," IEEE Transactions on Biomedical Engineering, vol 51(5), pp. 812-817, May 2004. 


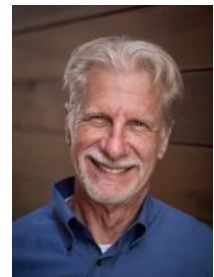

Matthew C. Smith (M'84-SM'91) is dual-degreed with a B.S. in electrical engineering and a B.S. in natural sciences from the University of South Florida (USF) in Tampa, FL in 1984. He received his M.S. in applied electromagnetics from the University of California San Diego, La Jolla, CA in 2019.

Currently he is a Ph.D. candidate at the University of California San Diego in the Applied Electromagnetics Group under Prof. Daniel Sievenpiper. Focused research areas include bioelectromagnetics, magnetic near field focusing arrays, underwater communications through magnetic induction and biophysics. He retired from Raytheon Space and Airborne Systems in El Segundo, CA in 2017. As a Sr. Engineering Fellow at Raytheon for over 25 years, he specialized in microwave and millimeter-wave circuits, subsystems and systems for radar, active and passive remote sensing, communications and electronic warfare systems. His design and technical experience include over fifty fielded and on-orbit microwave and millimeter-wave circuit designs, seven issued patents (two are NIST validated), and over twenty IEEE/SPIE papers from VHF to W-Band.

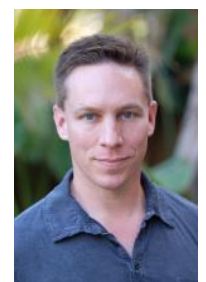

Daniel F. Sievenpiper (M'94-SM'04-F'09) received his B.S. and Ph.D. in electrical engineering from the University of California, Los Angeles, USA, in 1994 and 1999, respectively.

Dan joined HRL Laboratories in Malibu, CA in 1999. During the following 11 years, he and his team developed new electromagnetic structures with an emphasis on small, conformal, tunable, and steerable antennas. Dan held a variety of technical and management positions at HRL including Director of the Applied Electromagnetics Laboratory. In 2010 he joined UC San Diego as a professor in the Electrical and Computer Engineering Department, where his research is focused on artificial media, and the integration of active electronics with electromagnetic structures and antennas to enable new capabilities and applications. His recent work involves anisotropic and active metasurfaces, photonic and phononic topological insulators, small and wearable antennas, and biological applications of electromagnetics.

In 2008, Dan received the URSI Issac Koga Gold Medal, and also the IEEE Antennas and Propagation Society Piergiorgio Uslenghi Letters Prize Paper Award. In 2009, he was named as a Fellow of the IEEE, and in 2019, he was awarded the John D. Kraus Antenna Award. During 2010-2017, Dan served as an associate editor of IEEE Antennas and Wireless Propagation Letters. He also served as the chair of the IEEE Antennas and Propagation Society Administrative Committee on New Technology Directions from 2013-2014, and as the general chair of the IEEE Antennas and Propagation Symposium and URSI Radio Science Meeting which was held in San Diego in 2017. Beginning in 2019 he has served as the vice chair of the UCSD Electrical and Computer Engineering Department. Dan currently has more than 70 issued patents and more than 150 publications. 